\title{
Confined dynamical systems with Carroll and Galilei symmetries
}

\author{
Andrea Barducci* $^{*}$ and Roberto Casalbuoni ${ }^{\dagger}$ \\ Department of Physics, University of Florence and INFN Via G. Sansone 1, \\ 50019 Sesto Fiorentino (FI), Italy \\ Joaquim Gomis \\ Departament d'Estructura i Costituents de la Matèria and Departament de Física, \\ Universitat de Barcelona, Diagonal 647, 08028 Barcelona, Spain \\ and C.E.R. for Astrophysics, Particle Physics and Cosmology, Barcelona, Spain
}

(Received 1 October 2018; published 23 October 2018)

\begin{abstract}
We introduce a general method to construct, directly in configuration space, classes of dynamical systems invariant under generalizations of the Carroll and of the Galilei groups. The method does not make use of any nonrelativistic limiting procedure, although the starting point is a Lagrangian Poincaré invariant in the full space. It consists in considering a spacetime in $D+1$ dimensions and partitioning it in two parts, the first Minkowskian and the second Euclidean. The action consists of two terms that are separately invariant under the Minkowskian and Euclidean partitioning. One of those contains a system of lagrangian multipliers that confine the system to a subspace. The other term defines the dynamics of the system. The total lagrangian is invariant under the Carroll or the Galilei groups with zero central charge.
\end{abstract}

DOI: $10.1103 /$ PhysRevD.98.085018

\section{INTRODUCTION}

During the last few years, there has been interest in the "nonrelativistic" Carroll [1] and Galilei groups. One of the reasons for this interest is the relevance of BMS symmetry [2], which is related to the conformal Carroll group [3], to flat space holography [4-8]. Another reason is that nonrelativistic holography is a tool to study strongly interacting field theories in condensed matter systems $[9,10]$. The associated nonrelativistic gravities like Newton-Cartan [11], Horava [12], stringy Newton Cartan [13,14], torsional Newton Cartan [15] or Schrödinger [16], and Carroll [17-19] have been studied. There is matter coupled to nonrelativistic gravity, Carroll gravity, e.g., for particles [20-24], and for extended objects [14,21] for Galilean and for Carroll field theories [25,26] coupled to a NewtonCartan and Carroll [19] background.

Since most of the models invariant under the Carroll or Galilei group present in the literature are described by an action in phase space, the main purpose of this paper is to propose a general method to construct the corresponding

\footnotetext{
*barducci@fi.infn.it †casalbuoni@fi.infn.it "gomis@ecm.ub.es
}

Published by the American Physical Society under the terms of the Creative Commons Attribution 4.0 International license. Further distribution of this work must maintain attribution to the author(s) and the published article's title, journal citation, and DOI. Funded by SCOAP ${ }^{3}$. action in configuration space. As we shall see, this description corresponds to a system confined in a particular region of the spacetime. The corresponding Lagrangian systems will be invariant under generalizations of the Carroll and the Galilei groups, [23,27-32].

The Galilei and Carroll groups can be obtained via a convenient contraction of the Poincaré group [33]. In order to obtain the Bargmann group [34], that is the Galilei group with a central charge, it is necessary to extend the Poincaré group by a $U(1)$ factor, whereas, in the case of three dimensions, one needs to consider the contraction of Poincaré $\otimes U(1) \otimes U(1)$ [35], since in this case the Galilei group has two central extensions [36]. Otherwise one gets the Galilei algebra with zero central charge. It should be noted that the "nonrelativistic" limit is not unique $[27,28,37,38]$ when we have in mind extended objects. In other words, there is not a unique contraction of the Poincaré group. In some cases, these new algebras could contain noncentral extensions $[29,30]$. From now on, unless differently specified, for Galilei and Carroll algebras, we will mean the algebras with zero central or noncentral charges.

The general structure of the contracted algebras is that both contain the direct product of a Poincaré algebra in lower dimensions times an Euclidean algebra of rotations and translations in the complementary spatial dimensions. The contracted algebras contain also generalized boosts that rotate the generators of the lower Poincare with the ones of the Euclidean group. These boosts are a generalization of the ones of Galilei and Carroll groups. We will write the generators of the 
Poincaré group, $I S O(1, D), M_{\mu \nu}$ and $P_{\mu} \mu, \nu=0,1, \ldots, D$, as $\left(M_{\alpha \beta}, M_{a b}, P_{\alpha}, P_{a}, M_{\alpha b} \equiv B_{\alpha b}\right)$, with $\alpha, \beta=0,1, \ldots$, $k-1$ and $a, b=k, \ldots, D$.

The contractions will be defined dividing the components of the momentum $P_{\mu}$, in two sets $P_{\alpha}$ and $P_{a}$. The contractions we will consider consist, in the Carroll case, in rescaling the momenta $P_{\alpha}$ by a factor $1 / \omega$ and then taking the limit $\omega \rightarrow \infty$, explicitly $\tilde{P}_{\alpha}=P_{\alpha} / \omega$, where $\tilde{P}_{\alpha}$ will be the Carrollian generators. At the same time, we will need to rescale the boosts by the same factor. The same procedure is followed in the Galilei case, but this time we rescale the $P_{a}$ 's as $\tilde{P}_{a}=P_{a} / \omega$. The boosts work differently in the two cases. More precisely, in the Carroll case the commutator of the boosts with $P_{a}$ is proportional to $P_{\alpha}$, whereas in the Galilei case the two momenta are exchanged. This is the distinctive feature between the two contracted algebras.

An analogous procedure in spacetime can be followed by considering a Minkowski space-time $M(1, D)$, and partitioning it in two pieces, as the direct sum $M(1, k-1) \oplus$ $E(D+1-k)$, corresponding to the partition of the momentum generators considered above. In this case, we will consider the realization of the Poincare generators in terms of vector fields operating on the spacetime, $M(1, D)$, variables. Then, we will define two types of contractions by rescaling the relativistic coordinates $x^{\alpha}$ by a factor $\omega$, that is $\tilde{x}^{\alpha}=\omega x^{\alpha}$, in the Carroll case and, by the same factor $\omega$, the coordinates $x^{a}, \tilde{x}^{a}=\omega x^{a}$, in the Galilei case. The boosts connect the coordinates $x^{\alpha}$ to the $x^{a}$ 's for the Carroll contraction and the contrary happens for the Galilei one.

The previous procedure can be repeated, by varying $k, D$ times, not $D+1$, since rescaling all the momenta is equivalent to no rescaling. Notice that the commutation relations among the momenta and the generators of the Lorentz group are linear in the momenta, and, therefore, the commutation relations do not change for an overall rescaling of the momenta. Therefore, we may have $D$ contractions of the Carroll type and $D$ contractions of the Galilei type, in total $2 D$ possible contractions. We will define these as a $k$ contraction of the Carroll type, the contraction described above, and by the same rule, a contraction of the Galilei type. Notice that, in the previous literature, these contractions were called p-brane contractions.

A $k$ contraction of Carroll type and a $D+1-k$ contraction of Galilei type are dual one to the other. In fact, they can be obtained one from the other by exchanging the role of the boosts. In the case of $k=1$, this duality is close to the one considered in [24] although not quite the same.

Models invariant under the Carroll or the Galilei group have been previously obtained by taking convenient "nonrelativistic" limits on a relativistic action describing the original system in the $D+1$-dimensional Minkowski spacetime together with an appropriate rescaling of the parameters appearing in the action $[23,27-32,37,38]$. Some of these models without central extensions can be recovered by our approach.
Our general procedure in configuration space does not need to take any "nonrelativistic" limit to construct an invariant model under Carroll or Galilei. Starting with the Carroll case, we will first consider an action invariant under $\operatorname{ISO}(1, D)$, then we confine the system to a $M(1, k-1)$ subspace. The resulting action is automatically invariant under $I S O(1, k-1)$ and depends on the coordinates $x^{\alpha}$ only.

The model is trivially invariant under the Euclidean subalgebra and can be made invariant under the boosts adding convenient terms in the Euclidean sector of the total spacetime. Since the Carroll boosts map the Minkowski coordinates into the Euclidean ones, the corresponding variation of the action is linear in the Euclidean coordinates and in their derivatives. Therefore, we can construct compensating terms using Lagrange multipliers times the coordinates of the Euclidean space and their derivatives such to conserve the momentum also in that sector. We will be more specific in the following. In fact, the action might depend explicitly on the coordinates, but it needs to conserve the momentum. This allows to construct linear combinations of the Euclidean coordinates, We shall see that the Lagrangian multipliers of the time derivatives of the Euclidean coordinates can be thought as the canonical momenta of the Euclidean space variables. The variation induced by the boosts in the action describing the dynamical system is then compensated by a corresponding variation of the Lagrange multipliers. On the other hand, the equations of motion resulting from the variation of the Lagrange multipliers confine the system to live in the Minkowski subspace, since the typical implication is that the time derivatives of the Euclidean variables $x^{a}$ vanish confining the system in the Minkowski subspace and to not propagate in the Euclidean part. For this reason, we will call the part of the action relative to the Euclidean subspace as

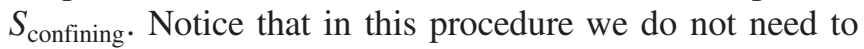
scale the parameters appearing in our Lagrangian. We will discuss in a detailed way the construction of discrete and continuous dynamical systems following what outlined before.

The same considerations can be made for dynamical systems invariant under the Galilei algebra. In fact, this time we will introduce an invariant action under the Euclidean group, $E(D+1-k)$, depending on the variables $x^{a}$ only. In this case, the Galilei boosts map the variables $x^{a}$ into the $x^{\alpha}$ 's. and we can get a model invariant under the full Galilei group, by the addition of a confining action consisting of Lagrange multipliers times convenient combinations of the $x^{\alpha}$ 's. Note that the mass-shell constraints of our models depend on momenta and coordinates on the longitudinal or on the transverse variables, but not depending on both. Several of the previously constructed models do depend on all the variables (see, e.g., [28,31,39]) and, therefore, cannot be obtained from our procedure. 
Strictly speaking, the Galilei invariant models obtained by this procedure are not dynamical in spacetime, since the action of these systems does not contain the time variable. This means that the dynamics happens only at a given time. In this sense, these models describe a sort of generalized instantons. The evolution in Euclidean space can be obtained in terms of a Euclidean coordinate and the associated Hamiltonian. In general, it is possible to obtain interesting results. For instance, considering the dual of the 1-contraction of the Carroll type (the Carroll particle), that is a $D$-contraction of the Galilei type, it is possible to obtain, in configuration space, a model equivalent to the one, obtained in [40] as the limit of a relativistic tachyon, in the phase space, for $c \rightarrow \infty$. This model has been called a Galilean massless particle [41], referring to the invariance under the Galilei algebra with zero central charge. This model describes an Euclidean nonrelativistic instanton.

The organization of the paper is as follows: in Sec. II, we discuss the $k$ contractions at the level of the algebra, whereas, in Sec. III, the same analysis is performed in configuration space. In Sec. IV, we present, in general, the construction of dynamical models invariant under Carroll and Galilei, in the discrete and in the continuous case, as, for instance, extended objects. In Sec. V, we will discuss various explicit examples of our procedure, ranging from the Carroll particle to massless particles up to a Carroll string. In the last section, we present our conclusions and discuss possible extensions of the present work.

\section{II. $\boldsymbol{k}$-CONTRACTIONS OF THE POINCARÉ GROUP}

Let us start considering the algebra of the Poincaré group in $D+1$ dimensions, $I S O(1, D)$

$$
\begin{aligned}
{\left[M_{\mu \nu}, M_{\rho \sigma}\right] } & =-i\left(\eta_{\mu \rho} M_{\nu \sigma}+\eta_{\nu \sigma} M_{\mu \rho}-\eta_{\mu \sigma} M_{\nu \rho}-\eta_{\nu \rho} M_{\mu \sigma}\right) \\
{\left[M_{\mu \nu}, P_{\rho}\right] } & =-i\left(\eta_{\mu \rho} P_{\nu}-\eta_{\nu \rho} P_{\mu}\right) \\
{\left[P_{\mu}, P_{\nu}\right] } & =0
\end{aligned}
$$

where $\mu=0,1, \ldots, D, \eta_{\mu \nu}=(-;+, \cdots,+)$.

Then, consider the following two subgroups of $I S O(1, D)$ : the Poincaré subgroup in $k$ dimensions, $I S O(1, k-1)$ and the Euclidean group of roto-translations in $D+1-k$ dimensions, generated, respectively, by

$$
\begin{array}{r}
I S O(1, k-1): M_{\alpha \beta}, \quad P_{\alpha}, \quad \alpha, \quad \beta=0,1, \ldots, k-1, \\
I S O(D+1-k): M_{a b}, \quad P_{a}, \quad a, b=k, \ldots, D .
\end{array}
$$

In these notations, the generators of $\operatorname{ISO}(1, D)$ are

$$
I S O(1, D): M_{\alpha \beta}, \quad M_{a b}, \quad P_{\alpha}, \quad P_{a}, \quad M_{\alpha b} \equiv B_{\alpha b} .
$$

The generators of $\operatorname{ISO}(1, k-1)$ satisfy the algebra (1), with the indices $\mu . \nu, \ldots$ replaced by $\alpha, \beta, \cdots$. Also the generators of $I S O(D+1-k)$ satisfy the algebra (1), with the indices $\mu . \nu, \ldots$ replaced by $a, b,, \cdots$ and with the replacement of the Minkowski metric with the Euclidean one, $\eta_{a b}=\delta_{a b}$. Furthermore, the two subalgebras commute. Now let us study the behaviour of the boosts under the two subalgebras. We have

$$
\begin{gathered}
I S O(1, k-1):\left[M_{\alpha \beta}, B_{\gamma c}\right]=-i\left(\eta_{\alpha \gamma} B_{\beta c}-\eta_{\beta \gamma} B_{\alpha c}\right), \\
{\left[B_{\alpha a}, P_{\beta}\right]=-i \eta_{\alpha \beta} P_{a},} \\
I S O(D+1-k):\left[M_{a b}, B_{\gamma c}\right]=-i\left(\eta_{a c} B_{\gamma b}-\eta_{b c} B_{\gamma a}\right), \\
{\left[B_{\alpha a}, P_{b}\right]=i \eta_{a b} P_{\alpha} .}
\end{gathered}
$$

We see that the boosts behave like vectors under both groups. Finally, the commutator among boosts is given by

$$
\left[B_{\alpha a}, B_{\beta b}\right]=-i\left(\eta_{\alpha \beta} M_{a b}+\eta_{a b} M_{\alpha \beta}\right),
$$

We will consider two types of contractions, rescaling in one case the momenta $P_{\alpha}$ and, in the other case, the momenta $P_{a}$. In both cases, the boosts will be rescaled. The first contraction is called a Carroll contraction [1,23,24,31] and it is obtained by the following rescaling, in the limit of $\omega \rightarrow \infty$

$$
\begin{array}{rlrl}
\tilde{M}_{\alpha \beta} & =M_{\alpha \beta}, & \tilde{P}_{\alpha} & =\frac{1}{\omega} P_{\alpha}, \\
\tilde{P}_{a} & =P_{a}, \quad \tilde{M}_{a b}=M_{a b}, \\
\tilde{B}_{\alpha a} & =\frac{1}{\omega} B_{\alpha a} .
\end{array}
$$

Notice that the commutators of $M_{\alpha \beta}$ and $M_{a b}$ with the momenta do not change, since they are linear in the momenta. Therefore, we will be interested only in the commutators of the new generators $\tilde{P}_{\alpha}, \tilde{P}_{a}$ and $\tilde{B}_{\alpha a}$. Using Eqs. (5)-(7), we find

$$
\left[\tilde{B}_{\alpha a}, \tilde{B}_{\beta b}\right]=0, \quad\left[\tilde{B}_{\alpha a}, \tilde{P}_{\beta}\right]=0, \quad\left[\tilde{B}_{\alpha a}, \tilde{P}_{b}\right]=i \eta_{a b} \tilde{P}_{\alpha} .
$$

We will denote the contracted algebra by $\mathcal{C}_{k}(1+D)$. Since we rescale the first $k$ momenta $P_{\alpha}$ we will call this contraction a " $k$-contraction" of the Carroll type. The other contraction that will be considered will be obtained by rescaling the $D+1-k$ momenta $P_{a}$ and this will be called a $D+1-k$ contraction of the Galilei type:

$$
\begin{array}{rlrl}
\tilde{M}_{\alpha \beta} & =M_{\alpha \beta}, & \tilde{P}_{\alpha}=P_{\alpha}, & \tilde{M}_{a b}=M_{a b}, \\
\tilde{P}_{a}=\frac{1}{\omega} P_{a}, & \tilde{B}_{\alpha a}=\frac{1}{\omega} B_{\alpha a} .
\end{array}
$$

Again, the interesting commutators are

$$
\left[\tilde{B}_{\alpha a}, B_{\beta c}\right]=0, \quad\left[\tilde{B}_{\alpha a}, \tilde{P}_{\beta}\right]=-i \eta_{\alpha \beta} P_{a}, \quad\left[\tilde{B}_{\alpha a}, \tilde{P}_{a}\right]=0 .
$$

This scaling is suggested by the nonrelativistic limit of relativistic branes $[27,29,32,37,38,42]$. The algebra obtained 
by this contraction will be denoted by $\mathcal{G}_{D+1-k}(1+D)$. The two algebras $\mathcal{C}_{k}(1+D)$ and $\mathcal{G}_{D+1-k}(1+D)$ are dual to each other; they go one into the other by exchanging the role of the momenta $P_{\alpha}$ and $P_{a}$ and of the metric tensors.

It should be noticed that this duality relating the Carroll and the Galilei contractions corresponds to quite different physical situations (see also in the following). In the case of the 1-contraction, a similar duality has been discussed in [24] although it is not quite the same.

\section{III. $\boldsymbol{k}$-CONTRACTIONS IN CONFIGURATION SPACE}

In this section, we will study the realization of the previous abstract algebras in a flat Minkowski spacetime, $M(1, D)$, of dimensions $D+1$, with coordinates and metric

$x^{\mu}, \quad \mu=0,1, \ldots, D, \quad \eta_{\mu \nu}=(-,+, \cdots,+)$.

Let us consider this space as the direct sum of two orthogonal subspaces, a Minkowski spacetime $M(1, k-1)$, of dimensions $k$, and an Euclidean space $E(D+1-k)$ of dimensions $D+1-k$ :

$$
M(1, D)=M(1, k-1) \oplus E(D+1-k) .
$$

The coordinates of the two subspaces will be chosen to agree with what was done in the previous section,

$$
x^{\alpha} \in M(1, k-1), \quad \alpha=0,1, \ldots, k-1,
$$

and

$$
x^{a} \in E(D+1-k), \quad a=k, k+1, \ldots, D .
$$

It is interesting to notice that this division of the spacetime is identical to the on used for defining a $k-1$ brane.

Therefore, as we did previously, we will define two commuting subgroups of the Poincare group in $D+1$ dimensions, $I S O(1, D)$. These are the Poincaré group in $k$ dimensions, $I S O(1, k-1)$ and the roto-translations group in $D+1-k$ dimensions, $I S O(D+1-k)$. Furthermore, there are the generators that mix together the two subgroups, that is the "boosts", $B_{\alpha a}$.

We will consider the Poincaré group $\operatorname{ISO}(1, D)$ generated by the following vector fields

$$
M_{\mu \nu}=-i\left(x_{\mu} \partial_{\nu}-x_{\nu} \partial_{\mu}\right), \quad P_{\mu}=-i \partial_{\mu}
$$

which satisfy the algebra (1).

According to the split of the spacetime illustrated before, the previous generators are split in

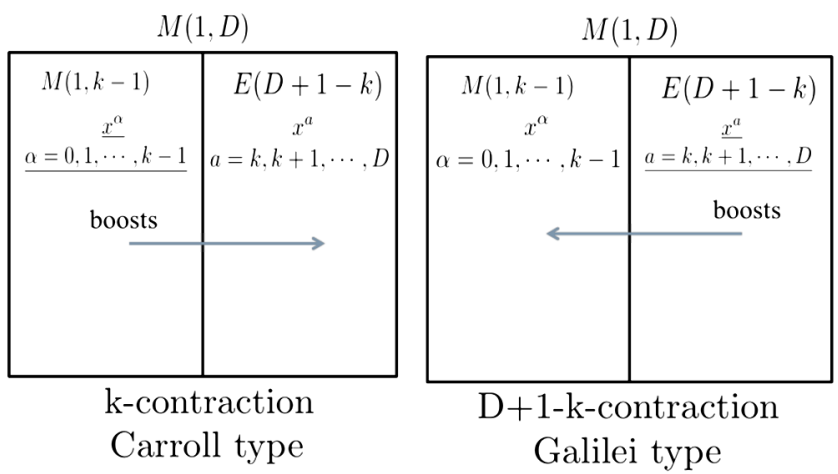

FIG. 1. The two types of contractions considered in the text. In the left panel, the Carroll type. In the right panel, the Galilei type. The rescaled variables are underlined. The arrows denote the directions in which the boosts act in the two cases.

$\left(M_{\alpha \beta}, P_{\alpha}\right) \in I S O(1, k-1), \quad\left(M_{a b}, P_{a}\right) \in I S O(D+1-k)$

and the "boosts"

$$
B_{\alpha a}=M_{\alpha a} .
$$

The contractions in configuration space are dual to the ones defined for the momenta (see, e.g., [31] for Carroll and $[29,30]$ for Galilei) as

$$
\begin{array}{llr}
\text { Carroll type, } & \tilde{x}^{\alpha}=\omega x^{\alpha}, & \tilde{x}^{a}=x^{a}, \\
\text { Galilei type }, & \tilde{x}^{\alpha}=x^{\alpha}, & \tilde{x}^{a}=\omega x^{a},
\end{array}
$$

in the limit $\omega \rightarrow \infty$.

These two types of contractions are not equivalent. The terminology used here derives from the case of 1contractions. In that case, the two contractions lead, respectively, to the Carroll and to the Galilei group with vanishing central charge (see, for instance, [33]). Therefore, we have a total of $2 D$ possible contractions For the case of p-branes, $p+1$ possible contractions have been considered [28].

On the other hand, the duality property considered in the previous section at the level of the exchange of the momenta for the two contracted groups is expressed here in terms of the exchange of the manifolds $M(1, k-1)$ and $E(D+1-k)$ (see Fig. 1).

In fact, the case in which all the variables are rescaled $(k=D+1)$, we get the original algebra. This is because the Lorentz generators do not change under rescaling of the coordinates, being homogeneous in the coordinates themselves. As for the momenta, since the commutation relations are linear in the momenta, they are left invariant by any common rescaling of the momenta. From this observation, it follows that the contractions 
Carroll type, $\quad \tilde{x}^{\alpha}=x^{\alpha}, \quad \tilde{x}^{a}=\frac{1}{\omega} x^{a}$,

Galilei type, $\quad \tilde{x}^{\alpha}=\frac{1}{\omega} x^{\alpha}, \quad \tilde{x}^{a}=x^{a}$,

give the same result as the ones following from (19).

Let us now consider explicitly the two cases:

Carroll type

Reexpressing the old variables in terms of the new ones, (19), we get

$$
\begin{aligned}
& M_{\alpha \beta}=-i\left(\tilde{x}_{\alpha} \tilde{\partial}_{\beta}-\tilde{x}_{\beta} \tilde{\partial}_{\alpha}\right)=\tilde{M}_{\alpha \beta}, \\
& M_{a b}=-i\left(\tilde{x}_{a} \tilde{\partial}_{b}-\tilde{x}_{b} \tilde{\partial}_{a}\right)=\tilde{M}_{a b}
\end{aligned}
$$

and, in the limit $\omega \rightarrow \infty$

$$
B_{\alpha a}=-i\left(\frac{1}{\omega} \tilde{x}_{\alpha} \tilde{\partial}_{a}-\omega \tilde{x}_{a} \tilde{\partial}_{\alpha}\right) \rightarrow \omega \tilde{B}_{\alpha a},
$$

where

$$
\tilde{B}_{\alpha a}=i \tilde{x}_{a} \tilde{\partial}_{\alpha}
$$

Furthermore,

$$
P_{\alpha}=-i \omega \tilde{\partial}_{\alpha}=\omega \tilde{P}_{\alpha},
$$

with

$$
\tilde{P}_{\alpha}=-i \tilde{\partial}_{\alpha}, \quad \tilde{P}_{a}=-i \tilde{\partial}_{a} .
$$

We see that this contraction coincides with the one of the previous section. Therefore, the commutation relations of the vector fields are the same obtained for the abstract generators of $\mathcal{C}_{k}(1+D)$.

Let us now consider the Galilei case:

\section{Galilei type}

Reexpressing the old variables in terms of the new ones, (19), we get

$$
\begin{aligned}
& M_{\alpha \beta}=-i\left(\tilde{x}_{\alpha} \tilde{\partial}_{\beta}-\tilde{x}_{\beta} \tilde{\partial}_{\alpha}\right)=\tilde{M}_{\alpha \beta}, \\
& M_{a b}=-i\left(\tilde{x}_{a} \tilde{\partial}_{b}-\tilde{x}_{b} \tilde{\partial}_{a}\right)=\tilde{M}_{a b}
\end{aligned}
$$

and

$$
B_{\alpha a}=-i\left(\omega \tilde{x}_{\alpha} \tilde{\partial}_{a}-\frac{1}{\omega} \tilde{x}_{a} \tilde{\partial}_{\alpha}\right) \rightarrow \omega \tilde{B}_{\alpha a}
$$

in the limit $\omega \rightarrow \infty$, with

$$
\tilde{B}_{\alpha a}=-i \tilde{x}_{\alpha} \tilde{\partial}_{a} .
$$

Furthermore

$$
P_{a}=-i \omega \tilde{\partial}_{a}=\omega \tilde{P}_{a},
$$

with

$$
\tilde{P}_{a}=-i \tilde{\partial}_{a}, \quad \tilde{P}_{\alpha}=-i \tilde{\partial}_{\alpha} .
$$

For the commutation relations, the considerations made in the Carroll case can be repeated here. In any case, for completeness, we repeat here the relevant commutators for the two cases:

Carroll type

$$
\left(\begin{array}{c|ccc} 
& B_{\beta b} & P_{\beta} & P_{b} \\
\hline B_{\alpha a} & 0 & 0 & i \eta_{a b} P_{\alpha} \\
P_{\alpha} & 0 & 0 & 0 \\
P_{a} & -i \eta_{a b} P_{\beta} & 0 & 0
\end{array}\right),
$$

Galilei type

$$
\left(\begin{array}{c|ccc} 
& B_{\beta b} & P_{\beta} & P_{b} \\
\hline B_{\alpha a} & 0 & -i \eta_{\alpha \beta} P_{a} & \\
P_{\alpha} & i \eta_{\alpha \beta} P_{a} & 0 & 0 \\
P_{a} & 0 & 0 & 0
\end{array}\right) .
$$

\section{BUILDING DYNAMICAL MODELS}

We will consider here the case of dynamical systems described by discrete variables and then by continuous variables. The first case corresponds to having a certain number of interacting pointlike objects. The second case corresponds to having extended objects as, for instance, branes. We will show how to construct models invariant either under Carroll or under Galilei. However, we will not consider here the case of field theories.

\section{A. Discrete models}

Let us start from the Carroll type of symmetry and consider a $k$-contraction. We suppose an action, describing $N$ interacting particles, invariant under a linear realization Poincaré group in $k$ dimensions:

$$
S_{P L}=\int d \tau L_{P L}\left(\dot{x}_{i}^{\alpha}, x_{i}^{\alpha}\right), \quad x_{i}^{\alpha} \in M(1, k-1),
$$

where the index $i=1, \ldots, N$ describes the type of particle. From the invariance under translations, $x_{i}^{\alpha} \rightarrow x_{i}^{\alpha}+a^{\alpha}$ it follows

$$
\sum_{i} q_{i \alpha}=0, \quad q_{i \alpha}=\frac{\partial L_{P L}}{\partial x_{i}^{\alpha}} .
$$

Introducing the canonical momenta,

$$
p_{i \alpha}=\frac{\partial L_{P L}}{\partial \dot{x}_{i}^{\alpha}}
$$




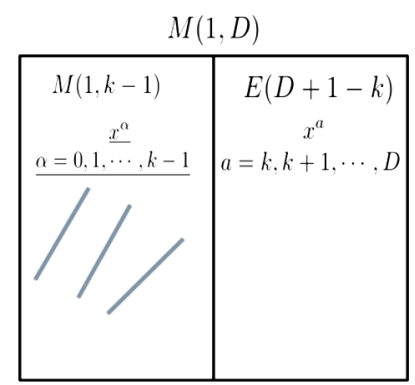

Point-like particles Carroll type

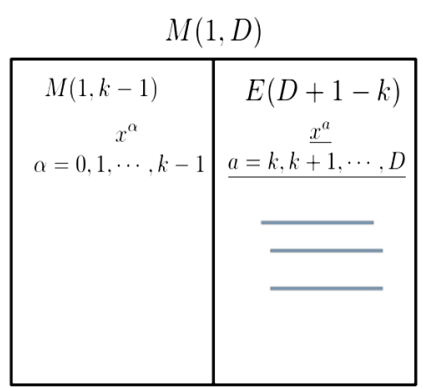

Point-like particles Galilei type
FIG. 2. The two types of pointlike systems considered in the text. In the left panel, the Carroll type. In the right panel, the Galilei type. The lines are a sketch of the world lines of particles living in the $M(1, k-1)$ Minkowski subspace (left panel) and of the world lines of the instantons living in the Euclidean subspace $E(D+1-k)$ (right panel).

the equations of motion are

$$
\dot{p}_{i \alpha}=q_{i \alpha},
$$

implying the conservation of the total momentum

$$
\dot{P}_{\alpha}=\sum_{i=1}^{N} \dot{p}_{i \alpha}=\sum_{i=1}^{N} q_{i \alpha}=0 .
$$

Now, let us consider the other part of the total spacetime $M(1, D)$, that is the Euclidean space $E(D+1-k)$. We will consider our point particles living only in the space $M(1, k-1)$ (see Fig. 2); therefore, we will introduce a set of constraints, confining the particles to stay in $M(1, k-1)$. Furthermore, if we want to implement the Carroll type of symmetry we have to require that this confinement action is invariant under $\operatorname{ISO}(D+1-k)$. In particular, we have to require invariance under translations. This can be realized introducing the center of mass coordinates in the Euclidean sector

$$
\bar{x}^{a}=\frac{1}{N} \sum_{i=1}^{N} x_{i}^{a} .
$$

Notice that any linear combination $\sum_{i}^{N} a_{i} x_{i}^{a}$, with $\sum_{i}^{N} a_{i}=1$, could also make the job. In order to get the momentum conservation, the confining action must depend only on the relative coordinates

$$
S_{\text {confining }}=\int d \tau \sum_{i=1}^{N}\left(\lambda_{a}^{i} \dot{x}_{i}^{a}+\mu_{a}^{i}\left(x_{i}^{a}-\bar{x}^{a}\right)\right) .
$$

Notice that we have the identity

$$
\sum_{i=1}^{N} \mu_{a}^{i}\left(x_{i}^{a}-\bar{x}^{a}\right)=\sum_{i=1}^{N}\left(\mu_{a}^{i}-\bar{\mu}_{a}\right) x_{i}^{a},
$$

where

$$
\bar{\mu}_{a}=\frac{1}{N} \sum_{i=1}^{N} \mu_{a}^{i}
$$

The equations of motion for the variables $x_{i}^{a}$ are

$$
\dot{p}_{i a}=\frac{d}{d \tau} \frac{\partial L}{\partial \dot{x}_{i}^{a}}=\frac{\partial L}{\partial x_{i}^{a}}=\left(\mu_{a}^{i}-\bar{\mu}_{a}\right),
$$

implying the conservation of the total momentum $P_{a}$ :

$$
\dot{P}_{a}=\sum_{i=1}^{N} \dot{p}_{i a}=0 .
$$

In this way, we enforce the translation invariance in $E(D+1-k)$. Furthermore, assuming that all the Lagrange multipliers transform like vectors under the rotation group $S O(D+1-k)$, the confining action satisfies all our requirements. The equations of motion resulting from this action are

$$
\dot{x}_{i}^{a}=0, \quad x_{a}^{i}=\bar{x}_{a} .
$$

We will now assume as total action

$$
S=S_{P L}+S_{\text {confining }}
$$

Since the two actions $S_{P L}$ and the confining one have separated variables, all the relations we have derived so far, continue to hold for the total action. Notice that the role of $S_{\text {confining }}$ is just to constrain the particles living in $M(1, k-1)$ to not escape from this space, they are "confined." We will show now that the total action has more symmetries than $\operatorname{ISO}(1, k-1) \otimes I S O(D+1-k)$. In fact, it is invariant under the Carroll algebra $\mathcal{C}_{k}(1+D)$. We have only to check the invariance under the boosts which are given by [compare with Eq. (23)]

$$
B_{\alpha}^{a}=-\sum_{i=1}^{N} x_{i}^{a} p_{i \alpha} .
$$

A boost with parameters $v_{a}^{\alpha}$ will generate the transformation

$$
\delta x_{i}^{\alpha}=\left\{\sum_{b \beta} v_{b}^{\beta} B_{\beta}^{b}, x_{i}^{\alpha}\right\}=v_{a}^{\alpha} x_{i}^{a}
$$

and

$$
\delta x_{i}^{a}=0,
$$

where we have used the Poisson brackets 


$$
\left\{x_{i}^{\alpha}, p_{\beta j}\right\}=\delta_{\beta}^{\alpha} \delta_{i j}
$$

Let us now evaluate the variation of the action $S$ under a Carroll boost. We have

$$
\delta S=\int d \tau \sum_{i=1}^{N}\left(p_{i \alpha} v_{a}^{\alpha} \dot{x}_{i}^{a}+q_{i \alpha} v_{a}^{\alpha} x_{i}^{a}+\delta \lambda_{a}^{i} \dot{x}_{i}^{a}+\delta \mu_{a}^{i}\left(x_{i}^{a}-\bar{x}^{a}\right)\right)
$$

This variation vanishes if

$$
\delta \lambda_{a}^{i}=-p_{i \alpha} v_{a}^{\alpha}, \quad \delta \mu_{a}^{i}=-q_{i \alpha} v_{a}^{\alpha} .
$$

Notice that from Eq. (37), due to the translational invariance of $S_{P L}$, it follows

$$
\sum_{i=1}^{N} \delta \mu_{a}^{i}=-\sum_{i=1}^{N} q_{i \alpha} v_{a}^{\alpha}=0,
$$

implying that also the variation of the term proportional to $\bar{x}^{a}$ is zero.

Therefore, the variations of the Lagrange multipliers $\lambda_{a}^{i}$ and $\mu_{a}^{i}$ are consistent with the translational invariance of the confining action, as it follows from (39). This is not accidental: the boost invariance of $S$ and the translation invariance of $S_{P L}$ and $S_{\text {confining }}$ are strictly related. In fact, since from Noether's theorem follows that continuous symmetries imply constants of motion, we see that if boosts and translational invariance in $E(D+1-k)$ are satisfied, then $S_{P L}$ must be translational invariant, as it follows from the commutation relations

$$
\left[B_{\alpha a}, P_{b}\right]=i \eta_{a b} P_{\alpha}
$$

and the fact that the commutator of two constants of motion is a constant of motion.

We can check directly that the boosts are conserved quantities using the equations of motion (44), (36) and (34)

$$
\frac{d}{d \tau} B_{\alpha a}=-\sum_{i=1}^{N} \dot{x}_{i}^{a} p_{i \alpha}-\sum_{i=1}^{N} x_{i}^{a} \dot{p}_{i \alpha}=-\sum_{i=1}^{N} \bar{x}^{a} q_{i \alpha}=0 .
$$

Our $S_{\text {confining }}$ action leads to the following primary constraints

$$
\phi_{i a}=p_{i a}-\lambda_{a}^{i}=0, \quad \pi_{\lambda_{a}^{i}}=0
$$

and $\pi_{\mu_{a}^{i}}=0$., since the action does not contain the time derivatives of the Lagrange multipliers. Of course, other constraints could arise from $S_{P L}$ The first constraint is consistent with the variation of the Lagrange multipliers under a boost. In fact,

$$
\delta p_{i a}=-\left\{\sum_{j=1}^{N} v_{b}^{\beta} x_{j}^{b} p_{j \beta}, p_{i a}\right\}=-v_{a}^{\alpha} p_{i \alpha}=\delta \lambda_{a}^{i} .
$$

The constraints (55) are second class, in fact

$$
\left\{\phi_{i a}, \pi_{\lambda_{b}^{j}}\right\}=-\delta_{i j} \delta_{a}^{b}
$$

Therefore, introducing Dirac brackets, $\{., .\}^{*}$, the Lagrange multipliers $\lambda_{a}^{i}$ and the momenta $p_{i a}$ can be identified, since for any dynamical variable, $A$, we have

$$
\left\{\lambda_{a}^{i}, A\right\}^{*}=\left\{p_{i a}, A\right\}^{*}
$$

To evaluate the canonical Lagrangian in the reduced phase space, where we have eliminated $\lambda_{a}^{i}$, we first notice that the terms $\lambda_{a}^{i} \dot{x}_{i}^{a}$ do not contribute to the Hamiltonian, being homogeneous of first degree in the time derivative. Therefore,

$$
L=\sum_{i=1}^{N}\left(p_{i \alpha} \dot{x}_{i}^{\alpha}+p_{i a} \dot{x}_{i}^{a}+\mu_{a}^{i}\left(x_{i}^{a}-\bar{x}^{a}\right)\right)-H_{P L},
$$

where $H_{P L}$ is the Hamiltonian evaluated from $L_{P L}$. If the Lagrangian implies some constraints as in the case of gauge invariance, e.g., invariance under diffeomorphisms, one needs to use the Dirac Hamiltonian. This action is boost invariant under the transformations

$\delta x_{i}^{\alpha}=v_{a}^{\alpha} x_{i}^{a}, \quad \delta x_{i}^{a}=0, \quad \delta p_{i a}=-v_{a}^{\alpha} p_{i \alpha}, \quad \delta p_{i \alpha}=0$,

with

$$
\delta \mu_{a}^{i}=v_{a}^{\alpha} \frac{\partial H_{P L}}{\partial x_{i}^{\alpha}} .
$$

The Galilei case, dual to the previous one, can be discussed exactly along the same lines. This time the action for the pointlike particles is defined in the Euclidean space dual to the Minkowski space of the Carroll case:

$$
S_{P L}=\int d \tau L\left(\dot{x}_{i}^{a}, x_{i}^{a}\right), \quad x_{i}^{a} \in E(D+1-k)
$$

and the confining term defined in $M(1, k-1)$

$$
S_{\text {confining }}=\int d \tau \sum_{i=1}^{N}\left(\lambda_{\alpha}^{i} \dot{x}_{i}^{\alpha}+\mu_{\alpha}^{i}\left(x_{i}^{\alpha}-\bar{x}^{\alpha}\right)\right) \text {, }
$$

with 


$$
\bar{x}^{\alpha}=\frac{1}{N} \sum_{i=1}^{N} x_{i}^{\alpha}
$$

Under boost we have the transformations

$$
\begin{aligned}
\delta x_{i}^{a} & =v_{\alpha}^{a} x_{i}^{\alpha}, \quad \delta x_{i}^{\alpha}=0, \quad \delta p_{i \alpha}=-v_{\alpha}^{a} p_{i \alpha}, \\
\delta p_{i a} & =0, \quad \delta \mu_{\alpha}^{i}=v_{\alpha}^{a} \frac{\partial H_{P L}}{\partial x_{i}^{a}}=0 .
\end{aligned}
$$

We will call the Carroll and the Galilei cases dual one to the other. A simple way to get this result, is to start with an action Poincaré invariant in the total space $M(1, D)$, say $S_{T}$ and define the actions in the two subspaces as

$\left(S_{P L}\right)_{\text {Carroll }}=\left.\left(S_{T}\right)\right|_{x^{a} \equiv 0}, \quad\left(S_{P L}\right)_{\text {Galilei }}=\left.\left(S_{T}\right)\right|_{x^{\alpha} \equiv 0}$.

In other words, the pointlike actions for the two cases are obtained by restricting the action $S_{T}$ to the two respective subspaces.

Of course, this is a quite general way of proceeding in order to get dual models. That is starting from an invariant action in the total space and restricting it to the two subspaces.

\section{B. Continuous models}

We would like to consider the dynamics of extended objects embedded in a confined region of the spacetime. We will show that the spacetime symmetries of these models are precisely the ones deriving from the $k$-contractions. Let us begin considering the Carroll type of contractions [1,23,24,31].

An extended object (for instance a brane) is mathematically described by mappings of a manifold (world sheet) to a target space. Let us start considering the target space as a flat spacetime in $D+1$ dimensions. Then, suppose to confine the extended object in the $M(1, k-1)$ Minkowski subspace of the spacetime. Assume that the world sheet is described by the coordinates $\left(\tau, \sigma_{i}\right)$, with $i=1,2, \ldots, m \leq k-1$. We will assume for this extended object an action invariant under a a linear representation of $\operatorname{ISO}(1, k-1)$

$$
S_{\mathrm{EO}}=\int_{V} d \tau \prod_{i=1}^{m} d \sigma_{i} \mathcal{L}\left(\dot{x}^{\alpha}, x_{, i}^{\alpha}\right) \equiv \int_{V} d \tau L_{\mathrm{EO}},
$$

with $x^{\alpha}$ the coordinates of the target space $M(1, k-1), V$ the volume which defines the system and $x_{, i}^{\alpha}=\partial x^{\alpha} / \partial \sigma_{i}$. Since we want to confine the extended object inside this space, we will add to this action a term keeping into account this condition

$$
\begin{aligned}
S_{\text {confining }} & =\int_{V} d \tau \prod_{i=1}^{m} d \sigma_{i}\left(\lambda_{a} \dot{x}^{a}+\sum_{j=1}^{m} \mu_{a}^{j} x_{, j}^{a}\right) \\
& \equiv \int d \tau L_{\text {confining }}
\end{aligned}
$$

where $x^{a}$ are the coordinates of the Euclidean target space, and the $\lambda$ 's and the $\mu$ 's are Lagrange multipliers. The aim of the confining term is to make vanish all the possible motions or vibrations of the extended object that could end in the space $E(D+1-k)$.

The total action is given by

$$
S=S_{\mathrm{EO}}+S_{\text {confining }} \equiv \int_{V} d \tau L .
$$

The confining term is invariant under the Euclidean group $I S O(D+1-k)$, assuming that the all Lagrange multipliers transform as vectors under the rotation group $S O(D+1-k)$. Let us define the following quantities

$$
\mathcal{P}_{\alpha}=\frac{\delta L_{\mathrm{EO}}}{\delta \dot{x}^{\alpha}}, \quad \mathcal{Q}_{\alpha}^{i}=\frac{\delta L_{\mathrm{EO}}}{\delta x_{, i}^{\alpha}}
$$

where $\mathcal{P}_{\alpha}$ is the momentum density. The equations of motion are

$$
\frac{\partial \mathcal{P}_{\alpha}}{\partial \tau}+\partial_{i} \mathcal{Q}_{\alpha}^{i}=0
$$

from which we have that the total momentum,

$$
P=\int_{V} \prod_{i=1}^{m} d \sigma_{i} \mathcal{P}_{\alpha}
$$

is conserved if,

$$
\left.\mathcal{Q}_{\alpha}^{i}\right|_{\Sigma=\partial V}=0
$$

on the boundary of the volume $V$.

Let us now show that this action is invariant under the boosts [see Eq. (23)] corresponding to a $k$-contraction of the Carroll type:

$$
\delta x^{\alpha}=v_{a}^{\alpha} x^{a}, \quad \delta x^{a}=0 .
$$

Let us evaluate the variation of the total Lagrangian under the previous transformations (the sum over the repeated indices is understood)

$$
\begin{aligned}
\delta L & =\int \prod_{i=1}^{m} d \sigma_{i}\left(\mathcal{P}_{\alpha} \delta \dot{x}^{\alpha}+\mathcal{Q}_{\alpha}^{i} \delta x_{, i}^{\alpha}+\delta \lambda_{a} \dot{x}^{a}+\delta \mu_{a}^{i} x_{, i}^{a}\right), \\
& =\int \prod_{i=1}^{m} d \sigma_{i}\left(\mathcal{P}_{\alpha} v_{a}^{\alpha} \dot{x}^{a}+\mathcal{Q}_{\alpha}^{i} v_{a}^{\alpha} x_{, i}^{a}+\delta \lambda_{a} \dot{x}^{a}+\delta \mu_{a}^{i} x_{, i}^{a}\right) .
\end{aligned}
$$


Therefore, the action is invariant by assuming the following transformation law for the Lagrange multiplier

$$
\delta \lambda_{a}=-\mathcal{P}_{\alpha} v_{a}^{\alpha}, \quad \delta \mu_{a}^{i}=-\mathcal{Q}_{\alpha}^{i} v_{a}^{\alpha}
$$

and in this way we have shown that this construction leads automatically to models invariant under the Carroll type of contracted groups considered in Sec. II. An analogous procedure can be made for the Galilei type.

Other features of these models that can be discussed before specifying $S_{E O}$ We have

$$
\mathcal{P}_{a}=\frac{\delta L_{\text {confining }}}{\delta \dot{x}^{a}}=\lambda_{a} .
$$

Since in the action the time derivatives of the Lagrange multipliers do not appear, it follows that the corresponding momenta vanish:

$$
\pi_{\lambda_{a}}=\frac{\delta S}{\delta \dot{\lambda}_{a}}=0
$$

These and the previous ones are second-class constraints. Introducing Dirac brackets, by definition we have

$$
\left\{\mathcal{P}_{a}, A\right\}^{*}=\left\{\lambda_{a}, A\right\}^{*},
$$

for any dynamical variable $A$. Therefore, in the reduced phase space, the momenta $\mathcal{P}_{a}$ and the Lagrange multipliers $\lambda_{a}$ can be identified. Notice that the same boosts generating the transformation (76) would generate the following variations for the momenta:

$$
\delta \mathcal{P}_{a}=-v_{a}^{\alpha} \mathcal{P}_{\alpha}, \quad \delta \mathcal{P}_{\alpha}=0,
$$

which is consistent with the variation of $\lambda_{a}$. The construction of the action in the reduced phase space goes as in the pointlike case.

Analogous models can be considered for the Galilei case. In this circumstance, we would take an action describing an extended object inside the Euclidean space $E(D+1-k)$

$$
S_{\mathrm{EO}}=\int_{V} d \tau \prod_{i=1}^{m} d \sigma_{i} \mathcal{L}\left(\dot{x}^{a}, x_{, i}^{a}\right) \equiv \int d \tau L_{\mathrm{EO}}
$$

with a confining term

$$
S_{\text {confining }}=\int_{V} d \tau \prod_{i=1}^{m} d \sigma_{i}\left(\lambda_{a}^{0} \dot{x}^{\alpha}+\sum_{j=1}^{m} \lambda_{a}^{j} x_{, j}^{\alpha}\right) \equiv \int d \tau L_{\text {confining }} .
$$

Then, one can repeat the same considerations made in the Carroll case. However, there is a very deep physical difference between the two cases, since the Carroll models

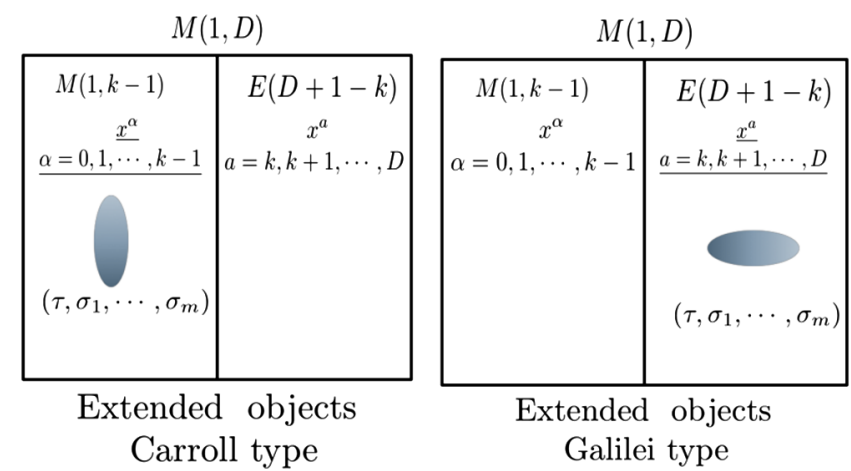

FIG. 3. The shaded figures are a sketch of the extended objects living in the $M(1, k-1)$ Minkowski subspace (left panel) and of the ones living in the Euclidean subspace $E(D+1-k)$ (right panel). The variables $\left(\tau, \sigma_{1}, \ldots, \sigma_{m}\right)$ describe the world sheet of the extended objects. We assume $m \leq k-1$ in the Carroll case and $m \leq D-k$ in the Galilei case.

are formulated in a Minkowski spacetime, whereas the Galilei case is formulated in an Euclidean space. As a consequence the Galilei case resembles the description of an instanton. Notice that the Carroll case corresponding to a $k$-contraction is dual to the $D+1-k$-contraction of Galilei type. However, when we use this duality to describe extended objects there are some conditions to be respected. In fact, we will assume that the dimensions of the world sheet of the extended object, $m+1$, are smaller or equal to the ones of the target space. Therefore, to have dual objects we have to require $m+1 \leq k$, in the Carroll case, and $m+1 \leq D+1-k$. Therefore, to have duality we need the condition (see Fig. 3)

$$
m \leq \min (k-1, D-k) .
$$

Also in the continuous case we can proceed as in the pointlike one, that is starting from an invariant action describing an extended object in $M(1, D)$ Minkowski space. Then, the action for Carroll is obtained by restricting this action to the space $M(1, k-1)$, that is by putting to zero the variables $x^{a}$. Analogously, in the Galilei case, one takes the restriction to $E(D+k-1)$, that is by putting to zero the variables $x^{\alpha}$. Of course, in order to get dual models, the condition (83) must be satisfied.

\section{EXAMPLES}

In this section, we will start from the description of a massive relativistic particle in the full spacetime $M(1, D)$ described by the invariant Diff action

$$
S=-M \int d \tau \sqrt{-\eta_{\mu \nu} \dot{x}^{\mu} \dot{x}^{\nu}}
$$

We will consider various examples. In particular, we will study, in the case of 1-contraction of the Carroll type, a 
massive particle and its Galilei dual, obtaining the Carroll particle studied in [23]. Its dual corresponds to the so called Galileian massless particle [40,41]. Notice that, here, massless refers to the fact that the corresponding representation of the Galilei algebra is with zero central charge. We will consider also a relativistic massless particle described by the action

$$
S=\int d \tau \frac{1}{2 e} \dot{x}^{\mu} \dot{x}^{\nu} \eta_{\mu \nu}
$$

\section{A. The Carroll massive particle}

We will consider the Carroll-type 1-contraction [1], using the action (84). The Minkowski spacetime reduces to a one-dimensional space described by the variable $x^{0}$. The total action is given by the restriction of (84) at this space, plus the confining term

$$
S=\int d \tau\left(-M \sqrt{\left(\dot{x}^{0}\right)^{2}}+\vec{\lambda} \cdot \dot{\vec{x}}\right) \equiv \int d \tau L .
$$

This action is invariant under diffeomorphisms in $\tau$. The momenta are given by

$\vec{p}=\frac{\partial L}{\partial \dot{\vec{x}}}=\vec{\lambda}, \quad p_{0}=-M \frac{\dot{x}^{0}}{\sqrt{\left(\dot{x}^{0}\right)^{2}}}=-M \epsilon\left(\dot{x}^{0}\right)$.

There is one first-class constraint

$$
\phi=p_{0}^{2}-M^{2}=0,
$$

and $2 D$ second-class constraints

$$
\phi_{i}=p_{i}-\lambda_{i}=0, \quad \psi_{i}=\pi_{\lambda_{i}}=0,
$$

with Poisson brackets

$$
\left\{\phi_{i}, \psi_{j}\right\}=-\delta_{i j} .
$$

Introducing Dirac brackets, one can eliminate the Lagrange multipliers $\vec{\lambda}$ in favour of the momenta $\vec{p}$. The action (86) is invariant under the Carroll boost transformations [see Eq. (23)] with parameters $\vec{v}$

$$
\delta x^{0}=\vec{v} \cdot \vec{x}, \quad \delta \vec{\lambda}=-\vec{v} p_{0}, \quad \delta \vec{x}=0 .
$$

In the reduced phase space, these transformations become

$$
\delta x^{0}=\vec{v} \cdot \vec{x}, \quad \delta \vec{x}=0, \quad \delta p_{0}=0, \quad \delta \vec{p}=-\vec{v} p_{0} .
$$

These transformations are generated by

$$
B_{0 a}=-x_{a} p_{0},
$$

corresponding to the vector fields of Eq. (23). The action in the reduced phase space is

$$
S=\int d \tau\left(\vec{p} \cdot \dot{\vec{x}}+p_{0} \dot{x}^{0}-\frac{e}{2}\left(p_{0}^{2}-M^{2}\right)\right) .
$$

This action is invariant under the boost transformations given in (92) and coincides with the phase space action studied in [23], where it has been obtained from the relativistic action (84) in the limit of zero light-velocity. From our point of view, the limit $c \rightarrow 0$ is equivalent to the Carroll 1-contraction of Eq. (19). Notice that the physical description of this particle corresponds to a massive particle in its rest-frame. In fact, from the variation of $\vec{\lambda}$, we get $\dot{\vec{x}}=0$.

\section{B. The Galilei particle}

The $D$-contracted Galilei case corresponds to the model described in the literature as the Galileian massless particle $[40,41,43]$. The action is obtained by the restriction of the action (84) to the Euclidean space $E(D)$ and adding the confining part. Notice that in order to get a real action we have changed the sign inside the square root and, for convenience, also the sign in front of it. Therefore, the action that we choose is

$$
\begin{aligned}
S & =\int d \tau\left(M \sqrt{\dot{x}^{a} \dot{x}^{b} \eta_{a b}}+\lambda \dot{x}^{0}\right) \\
& =\int d \tau\left(M \sqrt{\dot{\vec{x}}^{2}}+\lambda \dot{x}^{0}\right) \equiv \int d \tau L, \quad \eta_{a b}=\delta_{a b} .
\end{aligned}
$$

This action is invariant under diffeomorphisms in $\tau$; therefore, the canonical Hamiltonian vanishes identically. The momenta are given by

$$
\vec{p}=\frac{\partial L}{\partial \dot{\vec{x}}}=M \frac{\dot{\vec{x}}}{|\dot{\vec{x}}|}, \quad p_{0}=\frac{\partial L}{\partial \dot{x}^{0}}=\lambda .
$$

Therefore, there are three constraints,

$\phi=\vec{p}^{2}-M^{2}=0, \quad \phi_{1}=p_{0}-\lambda=0, \quad \phi_{2}=\pi_{\lambda}=0$,

since the time derivative of $\lambda$ does not appear in the action. We see that the first constraint is first-class, whereas the other two are second-class. In fact, their Poisson bracket is not zero

$$
\left\{\phi_{1}, \phi_{2}\right\}=-1 \text {. }
$$

Therefore, introducing the Dirac brackets in the reduced phase space, $p_{0}$ and $\lambda$ can be identified. This action is invariant under the boost transformations of the Galilei type generated by the Galilei boosts [see Eq. (28)], with parameters $v_{a}$. 


$$
\begin{gathered}
B_{a 0}=x_{0} p_{a}, \\
\delta \vec{x}=\vec{v} x^{0}, \quad \delta \lambda=-\vec{v} \cdot \vec{p} .
\end{gathered}
$$

In the reduced phase space, these transformations become

$$
\delta \vec{x}=\vec{v} x^{0}, \quad \delta \lambda=\delta p_{0}=-\vec{v} \cdot \vec{p},
$$

and the action is given by

$$
S=\int d \tau\left(\vec{p} \cdot \dot{\vec{x}}+p_{0} \dot{x}^{0}-\frac{e}{2}\left(\vec{p}^{2}-M^{2}\right)\right) .
$$

This action coincides with the one studied in $[40,41,43]$. The particle described by this model can be seen as a tachyon in the standard frame of its velocity, that is the frame where $\dot{x}^{0}=0$, as it follows varying $\lambda$ in the action. The model can also be obtained by the contraction in Eq. (20), in the limit $\omega \rightarrow \infty$ for a relativistic tachyon.

This is equivalent to consider the limit $c \rightarrow \infty$. Therefore, this particle exists at a single instant of time at any point of space. Since this model can be also obtained via Wick rotation on the action of a massive relativistic particle, technically it describes an instanton. Notice also that the condition $\dot{x}^{0}=0$ implies that the physical velocity $d \vec{x} / d x^{0} \rightarrow \infty$.

\section{A lightlike particle of the Galilei type}

Let us consider the Poincaré group in $D+1$ dimensions. If we introduce light cone variables, we can write the generators as

$$
\begin{aligned}
& P_{-}, \quad P_{+}, \quad P_{a}, \quad M_{-+}=M_{01}, \quad M_{a b}, \\
& B_{+a}, \quad B_{-a}, \quad a, b=2,3, \ldots, D,
\end{aligned}
$$

where

$$
P_{ \pm}=\frac{1}{2}\left(P^{0} \pm P^{1}\right), \quad B_{a}^{ \pm}=\left(B_{0 a} \pm B_{1 a}\right) .
$$

We can see that we have two subalgebras

$$
\mathcal{G}^{ \pm}:\left(M_{01}, P_{ \pm}, M_{a b}, P_{a}, B_{a}^{ \pm}\right) .
$$

These subalgebras have the property that leave invariant a null direction $n^{\mu}$, explicitly $\Lambda^{\mu}{ }_{\nu} n^{\nu}=\lambda n^{\mu}$. $\mathcal{G}^{+}$leaves invariant the direction $n_{+}^{\mu}=\delta_{+}^{\mu}$, whereas $\mathcal{G}^{-}$leaves invariant the direction $n_{-}^{\mu}=\delta_{-}^{\mu}$. In the case of $D+1=4$, these algebras are known as $I \operatorname{Sim}(2)^{ \pm}$. They are symmetries of the very special relativity [44] in place of the Poincaré group.
The relevant nonvanishing commutation relations are

$$
\begin{aligned}
{\left[B_{a}^{ \pm}, P_{ \pm}\right] } & =-i P_{a}, \quad\left[B_{a}^{ \pm}, M_{01}\right]= \pm i B_{a}^{ \pm}, \\
{\left[M_{01}, P_{ \pm}\right] } & = \pm i P_{ \pm} .
\end{aligned}
$$

In order to construct a a massless particle model, we consider a Galilean invariant model, it is useful to consider a $k=2$ contraction of the Poincaré group. Introducing the light-cone coordinates

$$
x_{ \pm}=x_{0} \pm x_{1},
$$

the generators of the contracted algebra in configuration space (see Sec. III) are

$$
\begin{aligned}
M_{01} & =-i\left(x_{+} \partial_{+}-x_{-} \partial_{-}\right), & P_{ \pm}=-i \partial_{ \pm}, \\
B_{a}^{ \pm} & =-i x_{ \pm} \partial_{a}=x_{ \pm} P_{a}, & \partial_{ \pm}=\frac{\partial}{\partial x_{ \pm}} .
\end{aligned}
$$

The transformation under the boosts $B_{a}^{ \pm}$are

$$
\delta x^{a}=-v_{ \pm}^{a} x_{ \pm}, \quad \delta x_{ \pm}=0 .
$$

In other words, the main feature of these contracted algebras is that they leave invariant one of the two branches of the light-cone, $x_{ \pm}=0$, respectively. Notice that the generator $M_{01}$ acts as a dilation operator on the light-cone variables $x_{ \pm}$. It follows that it leaves invariant one of the planes $x_{ \pm}=$constant. This suggests considering a massless particle in Minkowski space $M(1, D)$ given in (85) in the light-cone coordinates. Following the lines for a 1-contraction of the Galilei type, we will consider two possible actions for describing the particle in the $E(D-1)$ Euclidean space

$$
S_{ \pm}=\int d \tau\left(\frac{1}{2 e} \sum_{a=2}^{D} \dot{x}_{a}^{2}+\lambda_{ \pm} \dot{x}_{ \pm}\right) .
$$

These actions are invariant under the two subalgebras separately. We can check the invariance under the boosts $B_{a}^{ \pm}$. In fact, we have

$$
\delta S_{ \pm}=\int d \tau\left(-p_{a} v_{ \pm}^{a} \dot{x}_{ \pm}+\delta \lambda_{ \pm} x_{ \pm}\right) .
$$

Therefore, $S_{ \pm}$is invariant assuming

$$
\delta \lambda_{ \pm}=p_{a} v_{ \pm}^{a} .
$$

Let us notice that the two actions $S_{ \pm}$are invariant under the transformations generated by $M_{01}$. In fact, the corresponding transformations of $x_{ \pm}$are

$$
\delta x_{ \pm}=\mp s x_{ \pm},
$$


where $s$ is the infinitesimal parameter. The invariance follows assuming

$$
\delta \lambda_{ \pm}= \pm s \lambda_{ \pm}
$$

Also, this transformation is compatible with the identification of $\lambda_{ \pm}$with $p^{ \pm}$as it follows from the commutation relations given in Eqs. (106).

For simplicity let us now consider $S_{+}$. The same considerations will hold for $S_{-}$. The canonical momenta are, using light cone variables, we have the constraints

$p_{+}=\lambda_{+}, \quad p_{-}=0, \quad \vec{p}^{2}=0 \rightarrow \vec{p}=0, \quad \pi_{\lambda_{+}}=0$.

We have $D+1$ first class constraints and two second class constraints, with $2(D+2)$ degrees of freedom. Then, the model is described by 2 d.o.f, $p_{+}$and its conjugated coordinate.

The Dirac Hamiltonian is given by

$$
H_{D}=\frac{e}{2} p_{a}^{2}+\mu p_{-} .
$$

\section{A lightlike particle of the Carroll type}

We will consider now a lightlike particle within a 2contraction of the Carroll type. The action, in the Minkowski part of the spacetime, will depend on the two variables $x^{0}$ and $x^{1}$.

We start with the massless action in Eq. (85) restricted to the plane $\left(x^{0}, x^{1}\right)$ and we add the confining term:

$$
S=\int d \tau\left[\frac{1}{2 e}\left(\left(\dot{x}^{0}\right)^{2}-\left(\dot{x}^{1}\right)^{2}\right)+\lambda_{a} \dot{x}^{a}\right] .
$$

The canonical momenta are

$$
p_{0}=\frac{\dot{x}^{0}}{e}, \quad p_{1}=-\frac{\dot{x}^{1}}{e}, \quad p_{a}=\lambda_{a},
$$

from which we have the first class constraint

$$
\phi=p_{0}^{2}-p_{1}^{2}=0
$$

and 2(D-1) second class constraints

$$
\phi_{a}=p_{a}-\lambda_{a}, \quad \chi_{a}=\pi_{\lambda_{a}}=0, \quad\left\{\phi_{a}, \chi_{b}\right\}=-\delta_{a b} .
$$

As in the previous examples, in the reduced phase space (after using the second class constraints), we can identify $p_{a}$ with $\lambda_{a}$. We will assume the $\lambda_{a}$ 's transforming like vectors under the generators of $S O(D-1)$. The model is invariant under translations, and under the transformations generated by $M_{01}$, since this generates a Lorentz boost in the direction $D$ and it leaves invariant the quadratic form in the action. Furthermore, we have invariance under the two types of boosts $B_{0 a}$ and $B_{1 a}$ :

$$
\delta_{B_{0 a}} x^{0}=-v_{0 a} x^{a}, \quad \delta_{B_{0 a}} \lambda_{a}=p_{0} v_{0 a}
$$

and

$$
\delta_{B_{1 a}} x^{1}=-v_{1 a} x^{a}, \quad \delta_{B_{1 a}} \lambda_{a}=p_{1} v_{1 a} .
$$

The rest of the discussion goes as in the other examples. In this case, the Carroll particle moves in the plane $\left(x_{0}, x_{1}\right)$ at the speed of light.

In the case of the 2-contraction, we have reported only the case of a massless particle. We could as well to start with the action for a massive particle in $M(1, D)$. In this case, we would obtain for the Galilei particle the mass-shell conditions

$$
p_{0}^{2}-p_{1}^{2}=0, \quad \sum_{a} p_{a}^{2}=M^{2},
$$

that is a tachyon in $D+1$ dimensions, whereas for the Carroll case,

$$
p_{0}^{2}-p_{1}^{2}=M^{2},
$$

with considerations completely analogous to the ones discussed for the massless case.

\section{E. A model of two particles}

We start we considering the relativistic two- particle model [45]

$$
\begin{aligned}
L & =-\sqrt{-\left(m_{10}^{2}-V\left(r^{2}\right)\right) \dot{x}_{1}^{2}}-\sqrt{-\left(m_{20}^{2}-V\left(r^{2}\right)\right) \dot{x}_{2}{ }^{2}} \\
& =-\sum_{j=1,2} \sqrt{-m_{j}^{2}\left(r^{2}\right) \dot{x}_{j}^{2}},
\end{aligned}
$$

where $x_{j}(\tau),(j=1,2)$ are the spacetime coordinates of the two particles. $V\left(r^{2}\right)$ is any Poincaré invariant function of the squared relative distance $r^{2}=\left(x_{2}-x_{1}\right)^{2}, m_{j 0}$ 's are the rest masses of the particles and $m_{j}^{2}\left(r^{2}\right)=m_{j 0}^{2}-V\left(r^{2}\right)$ are the effective masses of the particles. The interaction breaks the individual invariance under diffeomorphism (Diff) of the action of two free particles, leaving a universal Diff invariance. The momenta are given by

$$
p_{i}=m_{i}^{2}\left(r^{2}\right) \frac{\dot{x}_{i}}{\sqrt{-m_{i}^{2}\left(r^{2}\right) \dot{x}_{i}^{2}}} .
$$

Following our procedure, the Carroll Lagrangian will be given by

$$
\begin{aligned}
L= & -\sqrt{-\left(m_{10}^{2}-V\left(r_{0}^{2}\right)\right)\left(\dot{x}_{1}^{0}\right)^{2}}-\sqrt{-\left(m_{20}^{2}-V\left(r_{0}^{2}\right)\right)\left(\dot{x}_{2}^{0}\right)^{2}} \\
& +\vec{\lambda}_{1} \cdot \dot{\vec{x}}_{1}+\vec{\lambda}_{2} \cdot \dot{\vec{x}}_{2}+\vec{\mu} \cdot \vec{r},
\end{aligned}
$$


where $r^{0}=x_{1}^{0}-x_{2}^{0}$. Like in the $k=1$ Carroll particle, the presence of the second class constraints allows to eliminate the $\lambda_{j}$ in terms of the momenta $p_{j}$. In the reduced space, the canonical action becomes

$S=\int d \tau\left(\sum_{j=1,2}\left(p_{i \mu} \dot{x}_{i}{ }^{\mu}-\frac{e_{i}}{2}\left(-p_{0 j}^{2}+m_{j}^{2}\left(r_{0}^{2}\right)\right)-\vec{\mu} \cdot \vec{r}\right)\right)$.

The particles do not move, although the momenta of the particles is not individually conserved. Notice that for these Carroll particles the momenta are not related to the velocities of the particles. This model is different from the two particle model of [23] where the two mass shell constraints depend on the total momenta of the particles.

The Galileian counterpart is given

$$
\begin{aligned}
L= & -\sqrt{\left(m_{10}^{2}-V\left(\vec{r}^{2}\right)\right) \dot{\vec{x}}_{1}^{2}}-\sqrt{\left(m_{20}^{2}-V\left(\vec{r}^{2}\right)\right) \dot{\vec{x}}_{2}^{2}} \\
& +\lambda_{1} \dot{x}_{1}^{0}+\mu\left(x_{1}^{0}-x_{2}^{0}\right)+\lambda_{2} \dot{x}_{2}^{0} .
\end{aligned}
$$

Like in the $k=1$ Galilei particle, the presence of the second class constraints allows to eliminate the $\lambda_{i}$ in terms of the momenta $p_{0 i}$. The action canonical in the reduced space is

$$
\begin{aligned}
S= & \int d \tau\left[\sum _ { i = 1 , 2 } \left(p_{0 i} \dot{x}_{i}^{0}+\vec{p}_{i} \cdot \vec{x}_{i}-\frac{e_{i}}{2}\left(\vec{p}_{i}^{2}-m_{j}^{2}\left(\vec{r}^{2}\right)\right)\right.\right. \\
& \left.-\mu\left(x_{1}^{0}-x_{2}^{0}\right) \cdot\right]
\end{aligned}
$$

The two particles described by this model can be seen as two tachyons in the standard frame of their velocity.

\section{F. 2-contraction for a Carroll string}

We recall the string action

$S_{\text {string }}=-T \int d \tau \int_{0}^{\pi} d \sigma \sqrt{\left(\dot{X} \cdot X^{\prime}\right)^{2}-(\dot{X})^{2}\left(X^{\prime}\right)^{2}}$.

Following what we have done in Sec. IV B, we assume the following action,

$$
S=S_{\text {string }}+\int d \tau \int_{0}^{\pi} d \sigma\left(\lambda_{a} \dot{X}^{a}+\mu_{a} X^{\prime a}\right),
$$

where, in the string action, $X^{\alpha}=\left(X^{0}, X^{1}\right)$, whereas $a=2,3, \ldots, D$. We get for the string Lagrangian density $\mathcal{L}_{\text {string }}$

$$
\mathcal{L}_{\text {string }}=-T \sqrt{\left(\dot{X}^{0} X^{1^{\prime}}-X^{0^{\prime}} \dot{X}^{1}\right)^{2}}
$$

The component of the canonical momentum are given by

$$
\begin{aligned}
& \mathcal{P}_{0}=-T \frac{\left(\dot{X}^{0} X^{1^{\prime}}-X^{0^{\prime}} \dot{X}^{1}\right)}{\sqrt{\left(\dot{X}^{0} X^{1^{\prime}}-X^{0^{\prime}} \dot{X}^{1}\right)^{2}}} X^{1^{\prime}}, \\
& \mathcal{P}_{1}=T \frac{\left(\dot{X}^{0} X^{1^{\prime}}-X^{0^{\prime}} \dot{X}^{1}\right)}{\sqrt{\left(\dot{X}^{0} X^{1^{\prime}}-X^{0^{\prime}} \dot{X}^{1}\right)^{2}}} X^{0^{\prime}}
\end{aligned}
$$

We get the primary constraints

$$
\mathcal{P}_{\alpha} X^{\alpha^{\prime}}=0, \quad \mathcal{P}^{2}+T^{2} X^{\prime 2}=0 .
$$

Let us evaluate the quantities $\mathcal{Q}_{\alpha}^{\sigma}=\partial \mathcal{L} / \partial X^{\prime \alpha}$

$$
\begin{gathered}
\mathcal{Q}_{0}^{\sigma}=T \frac{\left(\dot{X}^{0} X^{1^{\prime}}-X^{0^{\prime}} \dot{X}^{1}\right)}{\sqrt{\left(\dot{X}^{0} X^{1^{\prime}}-X^{0^{\prime}} \dot{X}^{1}\right)^{2}}} \dot{X}^{1} \\
\mathcal{Q}_{1}^{\sigma}=-T \frac{\left(\dot{X}^{0} X^{1^{\prime}}-X^{0^{\prime}} \dot{X}^{1}\right)}{\sqrt{\left(\dot{X}^{0} X^{1^{\prime}}-X^{0^{\prime}} \dot{X}^{1}\right)^{2}}} \dot{X}^{0} .
\end{gathered}
$$

The canonical action in the reduced space is given by

$$
\begin{aligned}
S= & \int d \tau d \sigma\left(\mathcal{P}_{1 \mu} \dot{X}_{1}^{\mu}+\mathcal{P}_{2 \mu} \dot{X}_{2}^{\mu}-\frac{e}{2}\left(\mathcal{P}_{\alpha} \mathcal{P}^{\alpha}+T^{2} X^{\prime 2}\right)\right. \\
& \left.-\mu\left(\mathcal{P}_{\alpha} X^{\prime \alpha}\right)-\mu_{a} X^{\prime a}\right) .
\end{aligned}
$$

This model is different from the Carroll string of [31]. Choosing the gauge conditions

$$
X^{0}=\tau, \quad X^{1}=\sigma,
$$

we have

$$
\mathcal{P}_{0}=-T, \quad \mathcal{P}_{1}=0, \quad \mathcal{Q}_{0}^{\sigma}=0, \quad \mathcal{Q}_{1}^{\sigma}=-T,
$$

and for the velocities

$$
\dot{X}^{0}=1, \quad \dot{X}^{1}=0 .
$$

These equations show that the string is at rest and, having fixed end points, it satisfies the Dirichlet boundary conditions. The total momentum is conserved

$P_{\alpha}=\int_{0}^{\pi} d \sigma \mathcal{P}_{\alpha}, \quad E=P^{0}=\pi T, \quad P_{1}=0$.

Therefore, in the case of a 2-contraction of the Carroll type, the string is the equivalent of the Carroll particle, that is the case of 1-contraction. For the considerations about the invariance under the boosts, one can repeat the general considerations made in Sec. IV B. 


\section{CONCLUSIONS AND OUTLOOK}

In this paper, we have introduced a general method to construct models with invariant Lagrangians under generalized Carroll or Galilei algebras (in the text called $k$-contractions). The method consists in starting from a spacetime in $D+1$ dimensions and partitioning it in two parts, the first Minkowskian and the second Euclidean. Then, a Carroll invariant model can be obtained by introducing a Minkowski invariant action in the first part of the spacetime, whereas in the second part a system of Lagrange multipliers, transforming in an appropriate way under the Euclidean group is introduced. This system is such that it compensates for the variations, induced by the Carroll boosts, of the action previously defined. The same procedure is done for the Galilei case, this time using a Lagrangian defined in the Euclidean sector and enlarging it with a system of Lagrange multipliers living in the first part of the spacetime. The main difference between Carroll and Galileian models constructed in this way, is that in the first case we have a real dynamical system, since the time coordinates are in the action, whereas in the second case the time variables appear only in the confined part, meaning that one is describing an instantonlike object.

This procedure could be generalized as follows: start with a target space (in the text the original spacetime), partitioned in two parts. Then, suppose that the target space supports a natural representation of some group $G$ (in the text the realization in terms of vector fields on the spacetime). Assume that the two sectors support natural representations of two groups $G_{I}$ and $G_{I I}$, which are both subgroups of $G$. Eventually assume that the Lie algebra of $G$ can be decomposed as follows:

$$
\text { Lie } \mathrm{G}=\mathrm{LieG}_{\mathrm{I}} \oplus \mathrm{LieG}_{\mathrm{II}} \oplus I \text {, }
$$

where $I$ are a set of intertwining generators mapping $\mathrm{LieG}_{\mathrm{I}}$ into $\mathrm{LieG}_{\mathrm{II}}$ or viceversa. Then, dynamical models describing systems, living in the target space, can be constructed following what we have done in the text. All these models would exhibit the confinement in one of the two sectors of the target space.

One could think to various possible extensions. For instance, one could describe a dynamical model with a generalized Galilei invariance, starting with a spacetime with two times, $M(2, D-1)$. Or else, starting from a Euclidean space $E(D+1)$, one could consider statistical systems confined to dimensions lower that $D+1$, but exhibiting a bigger symmetry. This approach could also be useful in theories with extra dimensions. We hope to be able to consider in the future some of these extensions in greater detail.

\section{ACKNOWLEDGMENTS}

We acknowledge Eric Bergshoeff, Jaume Gomis and Jorge Zanelli for comments. J. G. has been supported in part by Programa Nacional de Física de Partículas (FPA) 201346570-C2-1-P and Consolider Centro Nacional de Física de Partículas, Astropartículas y Nuclear (CPAN) and by the Spanish government (MINECO/FEDER) under project MDM-2014-0369 of ICCUB (Unidad de Excelencia María de Maeztu). J.G. has also been supported by CONICYT under grant PAI801620047 as a visiting professor of the Universidad Austral de Chile.
[1] J. M. Lévy-Leblond, Une nouvelle limite non-relativiste du group de Poincaré, Ann. Inst. H. Poincaré 3, 1 (1965); V. D. Sen Gupta, On an Analogue of the Galileo group, Nuovo Cimento 44, 512 (1966).

[2] H. Bondi, M. G. J. Van der Burg, and A. W. K. Metzner, Gravitational waves in general relativity. VII. Waves from axi-symmetric isolated systems, Proc. R. Soc. A 269, 21 (1962); R. K. Sachs, Gravitational waves in general relativity VIII. Waves in asymptotically flat space-time, Proc. R. Soc. A 270, 103 (1962).

[3] C. Duval, G. W. Gibbons, and P. A. Horvathy, Conformal Carroll groups, J. Phys. A 47, 335204 (2014); Conformal Carroll groups and BMS symmetry, Classical Quantum Gravity 31, 092001 (2014).

[4] T. Banks, A critique of pure string theory: Heterodox opinions of diverse dimensions, arXiv:hep-th/0306074.

[5] J. de Boer and S. N. Solodukhin, A Holographic reduction of Minkowski space-time, Nucl. Phys. B665, 545 (2003).
[6] G. Arcioni and C. Dappiaggi, Exploring the holographic principle in asymptotically flat space-times via the BMS group, Nucl. Phys. B674, 553 (2003).

[7] G. Barnich and C. Troessaert, Aspects of the BMS/CFT correspondence, J. High Energy Phys. 05 (2010) 062.

[8] A. Bagchi and R. Fareghbal, BMS/GCA redux: Towards flatspace holography from non-relativistic symmetries, J. High Energy Phys. 10 (2012) 092.

[9] S. Sachdev, Quantum Phase Transitions (Cambridge University Press, Cambridge, England, 2011).

[10] Y. Liu, K. Schalm, Y.-W. Sun, and J. Zaanen, Holographic Duality in Condensed Matter Physics (Cambridge University Press, Cambridge, England, 2015).

[11] E. Cartan, Sur les variétés à connexion affine et la théorie de la relativité généralisée. (première partie), Ann. Sci. Ecole Norm. Super. 40, 325 (1923).

[12] P. Horava, Quantum gravity at a Lifshitz point, Phys. Rev. D 79, 084008 (2009). 
[13] A. Bagchi and R. Gopakumar, Galilean conformal algebras and AdS/CFT, J. High Energy Phys. 07 (2009) 037.

[14] R. Andringa, E. Bergshoeff, J. Gomis, and M. de Roo, 'Stringy' Newton-Cartan gravity, Classical Quantum Gravity 29, 235020 (2012).

[15] M. H. Christensen, J. Hartong, N. A. Obers, and B. Rollier, Torsional Newton-Cartan geometry and Lifshitz holography, Phys. Rev. D 89, 061901 (2014).

[16] E. A. Bergshoeff, J. Hartong, and J. Rosseel, Torsional Newton-Cartan geometry and the Schrdinger algebra, Classical Quantum Gravity 32, 135017 (2015).

[17] M. Henneaux, Geometry of zero signature space-times, Bull. Soc. Math. Belg. 31, 47 (1979).

[18] J. Hartong, Gauging the Carroll algebra and ultra-relativistic gravity, J. High Energy Phys. 08 (2015) 069.

[19] E. Bergshoeff, J. Gomis, B. Rollier, J. Rosseel, and T. ter Veldhuis, Carroll versus Galilei gravity, J. High Energy Phys. 03 (2017) 165.

[20] K. Kuchar, Gravitation, geometry, and nonrelativistic quantum theory, Phys. Rev. D 22, 1285 (1980).

[21] J. Kluson, Canonical analysis of non-relativistic particle and superparticle, Eur. Phys. J. C 78, 117 (2018).

[22] A. Barducci, R. Casalbuoni, and J. Gomis, Non-relativistic spinning particle in a Newton-Cartan background, J. High Energy Phys. 01 (2018) 002.

[23] E. Bergshoeff, J. Gomis, and G. Longhi, Dynamics of Carroll particles, Classical Quantum Gravity 31, 205009 (2014).

[24] C. Duval, G. W. Gibbons, P. A. Horvathy, and P. M. Zhang, Carroll versus Newton and Galilei: Two dual non-Einsteinian concepts of time, Classical Quantum Gravity 31, 085016 (2014).

[25] K. Jensen, On the coupling of Galilean-invariant field theories to curved spacetime, SciPost Phys. 5, 011 (2018).

[26] J. Hartong, E. Kiritsis, and N. A. Obers, Schrödinger invariance from Lifshitz isometries in holography and field theory, Phys. Rev. D 92, 066003 (2015).

[27] J. Gomis and H. Ooguri, Nonrelativistic closed string theory, J. Math. Phys. (N.Y.) 42, 3127 (2001).

[28] C. Batlle, J. Gomis, and D. Not, Extended Galilean symmetries of non-relativistic strings, J. High Energy Phys. 02 (2017) 049.

[29] J. Brugues, T. Curtright, J. Gomis, and L. Mezincescu, Nonrelativistic strings and branes as non-linear realizations of Galilei groups, Phys. Lett. B 594, 227 (2004).
[30] J. Brugues, J. Gomis, and K. Kamimura, Newton-Hooke algebras, non-relativistic branes and generalized pp-wave metrics, Phys. Rev. D 73, 085011 (2006).

[31] B. Cardona, J. Gomis, and J. M. Pons, Dynamics of Carroll strings, J. High Energy Phys. 07 (2016) 050.

[32] J. Gomis, J. Gomis, and K. Kamimura, Non-relativistic superstrings: A new soluble sector of $\operatorname{AdS}(5)$ x $S^{5}$, J. High Energy Phys. 12 (2005) 024.

[33] H. Bacry and J. Levy-Leblond, Possible kinematics, J. Math. Phys. (N.Y.) 9, 1605 (1968).

[34] V. Bargmann, On Unitary ray representations of continuous groups, Ann. Math. 59, 1 (1954).

[35] E. A. Bergshoeff and J. Rosseel, Three-Dimensional Extended Bargmann Supergravity, Phys. Rev. Lett. 116, 251601 (2016).

[36] J.-M. Lévy-Leblond, in Galilei Group and Galilean Invariance, Group Theory and Applications (Loebl edition) (Acadamic Press, New York, 1972), Vol. II, p. 222.

[37] U.H. Danielsson, A. Guijosa, and M. Kruczenski, IIA/B, wound and wrapped, J. High Energy Phys. 10 (2000) 020.

[38] J. Gomis, K. Kamimura, and P. K. Townsend, Nonrelativistic superbranes, J. High Energy Phys. 11 (2004) 051.

[39] J. Gomis and F. Passerini, Rotating solutions of nonrelativistic string theory, Phys. Lett. B 617, 182 (2005).

[40] C. Batlle, J. Gomis, L. Mezincescu, and P. K. Townsend, Tachyons in the Galilean limit, J. High Energy Phys. 04 (2017) 120.

[41] J.-M. Souriau, Structure des syst'emes dynamiques (Dunod Editions, Paris, 1970) [C. H. Cushman-de Vries, Structure of Dynamical Systems: A Symplectic View of Physics, edited by R. H. Cushman and G. M. Tuynman (Birkhauser, Basel, Switzerland, 1997)].

[42] J. A. Garcia, A. Guijosa, and J. D. Vergara, A membrane action for WM2 / OM theory, Nucl. Phys. B630, 178 (2002).

[43] C. Duval and P. A. Horvathy, Non-relativistic conformal symmetries and Newton-Cartan structures, J. Phys. A 42, 465206 (2009).

[44] A. G. Cohen and S. L. Glashow, Very Special Relativity, Phys. Rev. Lett. 97, 021601 (2006).

[45] D. Dominici, J. Gomis, and G. Longhi, A Lagrangian for two interacting relativistic particles. 1, Nuovo Cimento B 48, 152 (1978). 